\title{
Correlation between HDL2, HDL3 and serum ferritin levels with fatty liver and NAFLD activity score (NAS) in liver histology of organ donors
}

Saman Nikeghbalian ${ }^{1}$, Rasoul Rahimi ${ }^{1,2^{*}}$, Hamed Nikoupour ${ }^{3}$, Neda Soleimani ${ }^{4}$, Sina Vakili ${ }^{5}$, Fatemeh Zal ${ }^{6}$ and Fahimeh Kaveh Baghbahadorani ${ }^{7}$

\begin{abstract}
Background: Nonalcoholic fatty liver disease (NAFLD) is one of the most important liver diseases. High-density lipoprotein $(\mathrm{HDL})$ has anti-atherogenic properties and its reduction can be associated with fatty liver. Serum ferritin levels are usually elevated in patients with NAFLD. This study aimed to evaluate the correlation between HDL subtypes and serum ferritin levels with evidence of NAFLD in liver histology of organ donors.

Methods: One hundred organ donor patients who were eligible for the study were included in the study and ferritin; HDL2 and HDL3 were measured in blood samples. Donated liver tissue biopsy specimens were evaluated for fatty liver and NAFLD activity score (NAS). In addition, AST and ALT were measured in recipients $24 \mathrm{~h}$ after transplant. All data abstracted and analyzed statistically.

Results: Serum HDL2 levels and HDL2/HDL3 ratio in patients with NAS $>1$ were significantly lower $(P<0.05)$. Serum levels of HDL3 and ferritin were not significantly associated with NAS $>1(P>0.05)$. In addition, serum ferritin $>1000$ $\mathrm{ng} / \mathrm{ml}$ in organ donors associated with increased AST and ALT levels $24 \mathrm{~h}$ after transplantation in the liver organ recipient.

Conclusions: Lower HDL2 values and HDL2/HDL3 ratio were associated with increased NAFLD activity score, but HDL3 and ferritin did not show such a relationship. In addition, higher levels of ferritin in organ donors may be associated with increased AST and ALT $24 \mathrm{~h}$ after liver transplantation in the organ recipient.
\end{abstract}

Keywords: High-density lipoprotein, Nonalcoholic fatty liver disease, Ferritin, Liver transplant

\section{Introduction}

Non-alcoholic fatty liver disease (NAFLD) is a form of hepatic steatosis in the absence of other causes of fat accumulation in the liver (such as heavy alcohol consumption) which is one of the most important liver diseases. Patients with NAFLD have hepatic steatosis with or without inflammation and fibrosis [1].

*Correspondence: rahimi.rasoul78@gmail.com

${ }^{2}$ Namazi Hospital, Shiraz University of Medical Sciences, Shiraz, Iran

Full list of author information is available at the end of the article
Patients with NAFLD may have mild to moderate increases in liver enzymes (AST and ALT), although normal aminotransferases do not rule out NAFLD. The prevalence of elevated aminotransferases among patients with NAFLD is unclear. The severity of elevated aminotransferases does not predict liver inflammation or fibrosis, and normal aminotransferases levels are not a sign of normal liver histology [2-4].

Fatty liver is associated with atherosclerosis and related diseases. Low HDL levels are associated with an increased risk of atherosclerosis [2, 4]. HDL levels are not only quantitatively important but also qualitatively 
important, and the combination of different HDL subtypes is associated with anti-atherogenic properties. Specifically, the amount of circulating HDL2 has the most protective effect against atherosclerosis [5]. In the clinic, two types of HDL can be measured in serum (HDL2 and HDL3). HDL2 particles are larger and richer in triglycerides, and HDL3 particles are smaller. Specifically, HDL2 is atheroprotective and has a higher percentage of unsaturated fatty acids. Some studies have shown that HDL2 is more decreased in patients with fatty liver, and HDL2 is a better predictor of cardiovascular disease than total HDL $[6,7]$.

In recent years, the association between elevated serum ferritin with metabolic syndrome and NAFLD has been suggested in many cases. Elevated ferritin levels have been observed in about $30 \%$ of patients diagnosed with NAFLD, which may be associated with elevated hepatic iron. Recent studies based on imaging with specific MRI protocols to detect iron levels in tissues have confirmed a close association between hepatocyte iron stores, steatosis, and metabolic disease $[5,8]$.

Nonalcoholic fatty liver disease (NAFLD) is an umbrella term for a range of liver conditions, some individuals with NAFLD can develop nonalcoholic steatohepatitis (NASH). NAFLD and NASH are actually two ends of the same spectrum. Steatosis is the accumulation of lipid droplets within hepatocytes and is considered pathologic when it affects more than 5\% of hepatocytes. The difference between NAFLD and NASH is based on histological findings $[9,10]$.

NAFLD activity score (NAS) is a system based on histological evaluation that covers all aspects of non-alcoholic fatty liver disease and can be effective in designing treatment in both children and adults. [11].

\section{Materials and methods}

We conducted the study between October 2019 and November 2020. This study was approved by the ethics committee of Shiraz University of Medical Sciences (ethics code: IR.SUMS.MED.REC.1399.328) and written informed consent was obtained from family of all participants in the study.

In this study, the relationship between serum levels of HDL2, HDL3 and ferritin with liver biopsy results (based on NAS) in organ donor patients was investigated. The study design was based on the possible role of lipid profile disorders and ferritin in NAFLD pathology. One of the main reasons for designing the study was the possibility of examining the liver biopsy in the donated organ.

Patients who were diagnosed as brain death and candidates for organ donation entered the study after fulfilling all the conditions for organ donation and if they met the inclusion criteria. The inclusion criteria in this study were: (1) Negative test results for HBV (hepatitis B virus), $\mathrm{HCV}$ (hepatitis $\mathrm{C}$ virus) and HIV (human immunodeficiency virus), (2) No previous history of liver cirrhosis or liver problems, (3) No previous history of hyperlipidemia. Exclusion criteria in this study was Evidence of mass or other liver disease on liver biopsy.

After reviewing the inclusion criteria, 100 patients who were diagnosed as brain death and candidate for organ donation were included in the study. Information about each patient, including age, sex, medical history, history of previous and current medications and the cause of brain death were recorded in a checklist for each patient. In this study, according to the specific conditions of organ donor brain death patients, BMI estimation was performed using mid upper arm circumference (MUAC). Table 1 shows how to estimate BMI using MUAC.

Before performing Harvest operation, $10 \mathrm{cc}$ of blood was taken to examine HDL subtypes and ferritin level. To measure the amounts of HDL2 and HDL3 in circulation, $1.5 \mathrm{cc}$ of serum was ultracentrifuged and the pattern of HDL subgroups was examined in a single step by adding heparin/Mn/DS reagent to simultaneously precipitate both the apoB-containing lipoproteins and HDL2. The reagent consisted of heparin $(1071 \mathrm{U} / \mathrm{ml}), \mathrm{MnCl} 2$ $(98.7 \mathrm{mg} / \mathrm{ml})$, and DS $(12 \mathrm{mg} / \mathrm{ml})$. The precipitation reagent $(0.06 \mathrm{ml})$ was added to $0.3 \mathrm{ml}$ of serum, mixed, left at room temperature for $30 \mathrm{~min}$, and centrifuged at $10,000 \mathrm{rpm}$ for $10 \mathrm{~min}$ at $4^{\circ}$ centigrade. An aliquot of the supernatant was taken for HDL3-C measurement. HDL-C in the supernatant was determined by HDL-C assay kit (parsazmoon, iran). The measured value for total HDL3-C was multiplied by 1.2 to correct for dilution by the reagents. The value for HDL2-C was calculated as the difference between the total HDL-C (directly determined in the serum by Parsazmoon kit) and HDL3C (All HDL assays were performed using Mindray BS200 auto-analyzer (China)). Serum ferritin was measured by Chemiluminescence immunoassay (CLIA) method. In addition, the same pathologist examined tissue biopsy of all donated livers and the fatty liver criteria and NAFLD Activity Score (NAS) were examined.

NAS scores range from 0 to 8 and do not include fibrosis. In fact, NAS include individual biopsy scores for steatosis (0-3), lobular inflammation $(0-3)$, and

Table 1 Estimate BMI using mid upper arm circumference (MUAC) [21]

\begin{tabular}{ll}
\hline Mid upper arm circumference (MUAC) (cm) & $\begin{array}{l}\text { Estimated body } \\
\text { mass index (BMI) } \\
\left(\mathbf{k g} / \mathbf{m}^{2}\right)\end{array}$ \\
\hline$<23.5$ & $<20$ \\
$>32.0$ & $>30$ \\
\hline
\end{tabular}


hepatocellular ballooning (0-2). A score of $0-2$ is seen in people who are not diagnosed with $\mathrm{NASH}$, a score of 3 to 4 includes people without NASH, people with borderline diagnosis and people diagnosed as $\mathrm{NASH}$, and a score of 5 to 8 includes those that considered as NASH [11].

Scoring in NAS is shown in Table 2.

To investigate the relationship between serum levels of HDL2, HDL3 and ferritin with NAS, all participants in the study were divided into two groups:

Group 1: cases with NAS $\leq 1$.

Group 2: cases with NAS $>1$.

In addition, in cases where the donated liver was suitable for transplantation and liver transplantation was performed, AST and ALT were measured in the liver recipient $24 \mathrm{~h}$ after receiving the organ and recorded in the relevant form.

Experimental results, pathology results of liver biopsy specimens and basic information were recorded in a checklist for each patient and finally analyzed by SPSS software. Mean \pm SD and frequency (relative frequency) were used to describe the quantitative and qualitative variables, respectively. Kolmogorov-Smirnov normality test and independent $t$ test were used to analyze the data (non-parametric test was used when necessary). Statistical analysis was performed using SPSS. v. 22. Statistical significance was defined as $P<0.05$.

\section{Results}

At the beginning of the study, One hundred organ donors were included in the study. Out of 100 donated liver organs, 89 were suitable for liver transplantation and transplanted to the appropriate recipients. Eleven donated livers were unsuitable for transplantation, six due to severe fatty liver and 5 due to necrosis. Of the 89 organ recipients, one died less than $24 \mathrm{~h}$ after receiving

Table 2 Scoring in NAFLD activity score (NAS) [22, 23]

\begin{tabular}{lll}
\hline Histological manifestations & Score & Criteria \\
\hline Steatosis & 0 & $<5 \%$ \\
& 1 & $5-33 \%$ \\
& 2 & $33-66 \%$ \\
Lobular inflammation & 3 & $>66 \%$ \\
& 0 & None \\
& 1 & $<2$ foci per 200X field \\
Ballooning & 2 & $2-4$ foci per 200X field \\
& 3 & $>4$ foci per 200X field \\
& 0 & None \\
& 1 & A few \\
& 2 & Present in many cells \\
\hline
\end{tabular}

the transplant. In the remaining 88 cases, AST and ALT were measured in recipients $24 \mathrm{~h}$ after transplantation.

Table 3 shows how BMI is distributed between the two groups.

Table 4 shows the demographic characteristics and values of HDL2, HDL3, and ferritin measured and statistical analysis in two groups.

As shown in Table 4, HDL2 values and HDL2/HDL3 ratio in group1 (NAS $\leq 1)$ were significantly higher $(P<0.05)$. There was no significant difference between the two groups in terms of age, HDL3 and ferritin.

In this study, the ferritin levels measured in organ donors were highly variable (120.3-3083.4). Therefore, in order to investigate the relationship between serum ferritin levels in organ donors and AST and ALT levels in organ recipients, ferritin levels were divided into two categories: $\leq 1000 \mathrm{ng} / \mathrm{ml}$ and $>1000 \mathrm{ng} / \mathrm{ml}$.

Table 5 shows the relationship between ferritin levels in organ donors and AST and ALT in 88 recipients $24 \mathrm{~h}$ after transplantation. The results showed that ferritin levels greater than $1000 \mathrm{ng} / \mathrm{ml}$ in organ donors were associated with an increase in AST and ALT levels $24 \mathrm{~h}$ after transplantation in the recipients.

Table 3 Body mass index (BMI) distribution between the two groups (group 1: NAS $\leq 1$, group 2: NAS $>1$ )

\begin{tabular}{lll}
\hline BMI $^{\mathbf{a}}\left(\mathbf{k g} / \mathbf{m}^{\mathbf{2}}\right)$ & $\begin{array}{c}\text { Group1: } \\
\mathbf{N}=\mathbf{7 7}\end{array}$ & $\begin{array}{l}\text { Group2: } \\
\mathbf{N}=\mathbf{2 3}\end{array}$ \\
\hline $\mathrm{BMI}<20$ & $24(31 \%)$ & $8(34 \%)$ \\
$\mathrm{BMI}=20-30$ & $51(66 \%)$ & $9(39 \%)$ \\
$\mathrm{BMI}>30$ & $2(2.6 \%)$ & $6(26 \%)$ \\
$\mathrm{N}$ & $77(100 \%)$ & $23(100 \%)$ \\
\hline
\end{tabular}

${ }^{a}$ Body mass index

${ }^{\mathrm{b}}$ Non-alcoholic fatty liver disease (NAFLD) activity score

Table 4 Demographic characteristics and measured values in the two groups (group 1: NAS $\leq 1$, group 2: NAS > 1)

\begin{tabular}{lccl}
\hline Parameter & $\begin{array}{c}\text { Group 1: NAS } \\
\mathbf{N}=\mathbf{7 7} \\
\text { Mean } \pm \text { SD }\end{array}$ & $\begin{array}{l}\text { Group 2: NAS }>\mathbf{1} \\
\mathbf{N}=\mathbf{2 3} \\
\text { Mean } \pm \text { SD }\end{array}$ & P value \\
\hline Age & $31.84 \pm 10.51$ & $32.95 \pm 211.73$ & 0.612 \\
HDL2 & $12.75 \pm 2.53$ & $7.62 \pm 2.36$ & $0.001^{*}$ \\
HDL3 & $22.61 \pm 4.77$ & $23.82 \pm 5.43$ & 0.329 \\
HDL2/HDL3 & $0.57 \pm 0.15$ & $0.22 \pm 0.07$ & $0.001^{*}$ \\
Ferritin & $1040.20 \pm 350.50$ & $1432.30 \pm 438.40$ & 0.103 \\
\hline
\end{tabular}

${ }^{*} P<0.05$ was considered statistically significant

${ }^{a}$ Non-alcoholic fatty liver disease (NAFLD) activity score 
Table 5 Relationship between ferritin levels in organ donor and liver transaminase levels in transplant recipients

\begin{tabular}{llll}
\hline Parameter & $\begin{array}{l}\text { Ferritin } \leq \mathbf{1 0 0 0} \mathbf{n g} / \mathbf{m l} \\
\mathbf{N}=\mathbf{6 1}\end{array}$ & $\begin{array}{l}\text { Ferritin }>\mathbf{1 0 0 0} \mathbf{n g} / \mathbf{m l} \\
\mathbf{N}=\mathbf{2 7}\end{array}$ & $\boldsymbol{P}$ value \\
\hline AST & $487.07 \pm 124.16$ & $911.45 \pm 314.23$ & $0.001^{*}$ \\
ALT & $518.41 \pm 168.31$ & $894.11 \pm 290.37$ & $0.012^{*}$ \\
\hline
\end{tabular}

${ }^{*} P<0.05$ was considered statistically significant

\section{Discussion}

NAFLD is the most common cause of chronic liver disease in Western societies and appears to be the most common indication for liver transplantation by 2030 [12].

Various studies have suggested the role of lipid profiles in the occurrence of NAFLD. In addition, some studies have suggested that HDL subtype values in NAFLD patients differ from those in the general population $[6,7]$.

We found a significant decrease in HDL2 and HDL2/ HDL3 ratio in patients with NAS $>1$, a finding similar to that other reports. Kantartzis et al. demonstrated that decreased HDL2 values and HDL2/HDL3 ratios were strongly correlated with fatty liver detection, which was much stronger than total HDL correlation. In this study, no difference in HDL3 values was observed between the two groups. However, liver biopsy was not used to diagnose NAFLD in this study [7].

In various studies, the diagnosis of NAFLD in patients has been associated with an increased risk of atherosclerosis [12, 13]. Arts et al. found that HDL2 and HDL3 levels decreased in cases of rheumatoid arthritis with atherosclerosis [13], while in the results of our study and Kantartzis et al. study, no difference was observed in the level of HDL3 in NAFLD patients.

In addition, Salonen et al. study showed that total HDL and HDL2 were inversely related to myocardial infarction but HDL3 was not significantly associated with it [14].

Normally most circulating HDL is in the form of HDL3 [15]. Similarly, in our study, circulating HDL3 levels were higher than HDL2. HDL particles are composed of 4 apolipoproteins. HDL subtypes are composed of lipoproteins Apo A-I and Apo A-II together or Apo A-I alone. HDL2 is often made of Apo A-I alone. Fadaei et al. demonstrated that Apo A-I levels are significantly lower in NAFLD patients [16]. This finding is similar to the results of our study that HDL2 levels were lower in patients with increased NAS.

Serum ferritin levels are usually elevated in patients with nonalcoholic fatty liver disease (NAFLD), due to inflammation or increased iron stores $[17,18]$. Kowdley et al. found that ferritin $>450 \mathrm{ng} / \mathrm{ml}$ was associated with higher levels of NAS, AST, ALT and increased hepatic fibrosis [17]. However, in our study, there was no relationship between serum ferritin levels in organ donors and NAS in liver biopsy. To interpret these results, we must consider the role of ferritin as an acute phase reactant in organ-donor brain death patients. This could be the reason for the very different amounts of ferritin in organ donors in this study.

Hagström et al. Showed that increased ferritin levels in NAFLD patients during long-term follow-up were associated with increased NAS and hepatic fibrosis [19].

As shown in Table 5, ferritin > $1000 \mathrm{ng} / \mathrm{ml}$ in organ donors was associated with increased AST and ALT $24 \mathrm{~h}$ after transplantation in recipients. This may be related to the primary inflammatory process that increases ferritin in the organ donor.

Wakiya et al. demonstrated that serum ferritin $>1000$ $\mathrm{ng} / \mathrm{ml}$ in organ donors was associated with increased intraoperative ferritin levels in the recipient. In addition, in this study, serum ferritin $>1000 \mathrm{ng} / \mathrm{ml}$ in transplant recipients was associated with longer warm ischemia time and increased levels of hepatic transaminases after surgery. Finally, in this study, increased ferritin levels in organ donors were introduced as a predictor of ischemia/ reperfusion injury in donated liver [20]. These findings are similar to the results of our study that elevated Ferritin levels in donors were associated with increased hepatic transaminases in recipients.

This study has been associated with limitations. One of the limitations was the impossibility of examining various factors in the incidence of fatty liver (due to the special conditions of patients diagnosed with brain death). On the other hand, brain dead patients are prone to increased ferritin as an inflammatory response that can affect their ferritin levels and act as a data-influencing factor.

Finally and based on our results, we can introduce HDL2 and HDL2/HDL3 as two contributing factors to predict the quality of liver pathology, especially in the case of NAFLD in organ donors. In addition, due to the limitations of liver biopsy for the diagnosis of NAFLD in the general population, it may be possible to use HDL2 and HDL3 measurements to diagnose NAFLD.

Although no correlation was found between serum ferritin levels and the pathology of liver biopsy in our study, the association between elevated ferritin in donors and elevated liver transaminases in recipients could confirm the role of ferritin as a predictor of liver damage.

\section{Conclusion}

Taken together, these data recommend that HDL subtypes can play an important role in predicting fatty liver. Measuring HDL2 and HDL3 is an easy and accessible way to predict the quality of liver pathology in donors. This method 
can also be used to predict fatty liver in the general population. In addition, ferritin levels in liver donors may be used to predict liver damage and transaminase levels in transplant recipients. Although considering the disparate findings above, additional extensive studies are needed to better examine the relationship between HDL2, HDL3, serum ferritin, liver transaminases, and liver pathologies.

\section{Abbreviations}

NAFLD: Nonalcoholic fatty liver disease; HDL: High-density lipoprotein; AST: Aspartate aminotransferase; ALT: Alanine transaminase; NASH: Nonalcoholic steatohepatitis; NAS: Nonalcoholic fatty liver disease activity score; VLDL: Very low density lipoproteins; LDL: Low-density lipoproteins; BMI: Body mass index; MUAC: Mid upper arm circumference; Apo: Apolipoprotein.

\section{Acknowledgements}

The authors would like to thank Shiraz University of Medical Sciences, Shiraz, Iran.

\section{Authors' contributions}

$\mathrm{SN}, \mathrm{RR}, \mathrm{NS}, \mathrm{FK}, \mathrm{HN}$ aided in the conceptualization, design, and critical revision of the final manuscript. SN, RR, NS, SV, FZ aided in design, preparation of manuscript and critical revision of the final manuscript SN, RR, FK, HN aided in data analysis and critical revision of the final manuscript. All authors read and approved the final manuscript.

\section{Funding}

The study was funded by Shiraz University of Medical Sciences (Grant \# 21083).

\section{Availability of data and materials}

All respectable readers and researchers can request the data by directly contacting the primary author at rahimi.rasoul78@gmail.com.

\section{Declarations}

\section{Ethics approval and consent to participate}

The trial protocol had been approved by the Medical Ethics Committee of Shiraz University of Medical Sciences (ethics code: IR.SUMS.MED.REC.1399.328) and written informed consent was obtained from family of all participants in the study.Declarationof Helsinki: All methods were performed in accordance with the relevant guidelines and regulations.

\section{Consent for publication}

Not applicable.

\section{Competing interests}

The authors declare that they have no competing interests.

\section{Author details}

${ }^{1}$ Department of Hepatobiliary and Transplantation Surgery, Shiraz Organ Transplant Center, Shiraz University of Medical Sciences, Shiraz, Iran. ${ }^{2}$ Namazi Hospital, Shiraz University of Medical Sciences, Shiraz, Iran. ${ }^{3}$ Shiraz University of Medical Sciences, Shiraz, Iran. ${ }^{4}$ Department of Pathology, School of Medicine, Shiraz University of Medical Sciences, Shiraz, Iran. ${ }^{5}$ Biochemistry Department, Medical School, Shiraz University of Medical Sciences, Shiraz, Iran. ${ }^{6}$ Department of Biochemistry, Shiraz University of Medical Sciences, Shiraz, Iran. ${ }^{7}$ Maternal-Fetal Medicine Research Center, Shiraz University of Medical Sciences, Shiraz, Iran.

Received: 14 May 2021 Accepted: 8 October 2021

Published online: 27 October 2021

\section{References}

1. Williams CD, Stengel J, Asike MI, Torres DM, Shaw J, Contreras M, Landt $\mathrm{CL}$, Harrison SA. Prevalence of nonalcoholic fatty liver disease and nonalcoholic steatohepatitis among a largely middle-aged population utilizing ultrasound and liver biopsy: a prospective study. Gastroenterology. 2011;140(1):124-31.

2. Cohen JA, Kaplan MM. The SGOT/SGPT ratio--an indicator of alcoholic liver disease. Dig Dis Sci. 1979;24(11):835-8.

3. Falck-Ytter Y, Younossi ZM, Marchesini G, McCullough AJ. Clinical features and natural history of nonalcoholic steatosis syndromes. Semin Liver Dis. 2001;21(1):17-26.

4. McCullough AJ. The clinical features, diagnosis and natural history of nonalcoholic fatty liver disease. Clin Liver Dis. 2004;8(3):521-33.

5. Haap M, Machann J, von Friedeburg C, Schick F, Stefan N, Schwenzer $N F$, Fritsche A, Häring HU, Thamer C. Insulin sensitivity and liver fat: role of iron load. J Clin Endocrinol Metab. 2011;96(6):E958-961.

6. Kajiyama G, Takata K, Horiuchi I, Nakagawa M, Yamamoto M, Miyoshi A. Changes in high density lipoproteins in patients with hepatobiliary diseases. Levels and lipid composition of HDL2 and HDL3 and LCAT reaction. Gastroenterol Jpn. 1983;18(2):98-108.

7. Kantartzis K, Rittig K, Cegan A, Machann J, Schick F, Balletshofer B, Fritsche A, Schleicher E, Häring HU, Stefan N. Fatty liver is independently associated with alterations in circulating HDL2 and HDL3 subfractions. Diabetes Care. 2008;31(2):366-8.

8. Dongiovanni P, Fracanzani AL, Fargion S, Valenti L. Iron in fatty liver and in the metabolic syndrome: a promising therapeutic target. J Hepatol. 2011;55(4):920-32.

9. Brunt EM. Alcoholic and nonalcoholic fatty liver disease. In: Odze RD, Goldblum JR, editors. Surgical pathology of the Gl tract, liver, biliary tract and pancreas. Elsevier; 2009.

10. Kleiner DE, Brunt EM. Nonalcoholic fatty liver disease: pathologic patterns and biopsy evaluation in clinical research. Semin Liver Dis. 2012;32(1):3-13.

11. Brunt EM. Nonalcoholic steatohepatitis: definition and pathology. Semin Liver Dis. 2001;21(1):3-16.

12. Byrne CD, Targher G. NAFLD: a multisystem disease. J Hepatol. 2015;62(1 Suppl):S47-64.

13. Arts E, Fransen J, Lemmers H, Stalenhoef A, Joosten L, van Riel P, Popa CD. High-density lipoprotein cholesterol subfractions HDL2 and HDL3 are reduced in women with rheumatoid arthritis and may augment the cardiovascular risk of women with RA: a cross-sectional study. Arthritis Res Ther. 2012;14(3):R116.

14. Salonen JT, Salonen R, Seppänen K, Rauramaa R, Tuomilehto J. HDL, HDL2, and HDL3 subfractions, and the risk of acute myocardial infarction. A prospective population study in eastern Finnish men. Circulation. 1991;84(1):129-39.

15. Sich D, Saïdi Y, Giral P, Lagrost L, Egloff M, Auer C, Gautier V, Turpin G, Beucler I. Hyperalphalipoproteinemia: characterization of a cardioprotective profile associating increased high-density lipoprotein2 levels and decreased hepatic lipase activity. Metabolism. 1998;47(8):965-73.

16. Fadaei R, Poustchi H, Meshkani R, Moradi N, Golmohammadi T, Merat S. Impaired HDL cholesterol efflux capacity in patients with non-alcoholic fatty liver disease is associated with subclinical atherosclerosis. Sci Rep. 2018;8(1):11691.

17. Kowdley KV, Belt P, Wilson LA, Yeh MM, Neuschwander-Tetri BA, Chalasani N, Sanyal AJ, Nelson JE. Serum ferritin is an independent predictor of histologic severity and advanced fibrosis in patients with nonalcoholic fatty liver disease. Hepatology. 2012;55(1):77-85.

18. Trombini P, Piperno A. Ferritin, metabolic syndrome and NAFLD: elective attractions and dangerous liaisons. J Hepatol. 2007;46(4):549-52.

19. Hagström H, Nasr P, Bottai M, Ekstedt M, Kechagias S, Hultcrantz R, Stål P. Elevated serum ferritin is associated with increased mortality in non-alcoholic fatty liver disease after 16 years of follow-up. Liver Int. 2016;36(11):1688-95

20. Wakiya T, Sanada Y, Urahashi T, Ihara Y, Yamada N, Okada N, Hirata Y, Hakamada K, Yasuda Y, Mizuta K. Impact of the serum ferritin concentration in liver transplantation. Liver Transpl. 2015;21(11):1419-27. 
21. Darnis S, Fareau N, Corallo CE, Poole S, Dooley MJ, Cheng AC. Estimation of body weight in hospitalized patients. QJM. 2012;105(8):769-74.

22. Brunt EM, Kleiner DE, Wilson LA, Belt $P$, Neuschwander-Tetri BA. Nonalcoholic fatty liver disease (NAFLD) activity score and the histopathologic diagnosis in NAFLD: distinct clinicopathologic meanings. Hepatology. 2011;53(3):810-20.

23. Kleiner DE, Brunt EM, Van Natta M, Behling C, Contos MJ, Cummings OW, Ferrell LD, Liu YC, Torbenson MS, Unalp-Arida A, et al. Design and validation of a histological scoring system for nonalcoholic fatty liver disease. Hepatology. 2005;41(6):1313-21.

\section{Publisher's Note}

Springer Nature remains neutral with regard to jurisdictional claims in published maps and institutional affiliations.
Ready to submit your research? Choose BMC and benefit from:

- fast, convenient online submission

- thorough peer review by experienced researchers in your field

- rapid publication on acceptance

- support for research data, including large and complex data types

- gold Open Access which fosters wider collaboration and increased citations

- maximum visibility for your research: over $100 \mathrm{M}$ website views per year

At BMC, research is always in progress.

Learn more biomedcentral.com/submissions 